\title{
Circularidades económicas y observación de segundo orden: La realidad de las calificaciones crediticias
}

\author{
Economic Circularities and Second-Order Observation: The Reality \\ of Ratings
}

Elena Esposito

Departamento de Comunicación y Economía, Universidad de Módena y Reggio

Emilia, Italia

\section{RESUMEN}

¿Pueden los observadores observar la economía desde el exterior? Desarrollos recientes en la sociología económica tienden a desdibujar la clásica distinción y combinación entre economía y sociedad, y a avanzar hacia un modelo en el que el observador se encuentra necesariamente dentro de la sociedad que él describe. El comportamiento de los agentes financieros se puede analizar mediante la combinación de dos conceptos: el concurso de belleza y el riesgo moral -una combinación que permite una traducción de su comportamiento en los términos y tradición de la teoría de la observación. El concurso de belleza de Keynes puede ser interpretado como un reconocimiento sistemático de la observación de segundo orden: los operadores financieros observan principalmente a otros observadores y lo que estos observan. Esta observación produce circularidades peculiares -incluyendo el insoluble problema del riesgo moral, el cual reproduce el famoso modelo de Merton de las profecías autocumplidas y autofrustradas en el campo de las finanzas. Sin embargo, si las finanzas consisten en observaciones de segundo orden, entonces sus movimientos no pueden ser explicados más con referencia al mundo, sino con referencia a la observación y sus estructuras: la referencia a la realidad de las finanzas es proporcionada crecientemente por calificaciones crediticias que solo pueden ofrecer información respecto de lo que los demás observan. La expansión de las calificaciones crediticias en las últimas décadas y las dudas sobre su fiabilidad están relacionadas con el movimiento generalizado de la sociedad moderna hacia la observación de segundo orden, lo cual produce problemas y enigmas específicos, así como estructuras y restricciones.

PALABRAS ClaVE: Teoría de la Observación; Incrustación; Performatividad; Calificaciones Crediticias; Finanzas; Concurso de Belleza, Riesgo Moral

\section{ABSTRACT}

Can observers observe the economy from the outside? Recent developments in economic sociology tend to blur the classic distinction and combination of economy and society and to move to a model in which the observer is necessarily inside the society he describes. The behavior of financial actors can then be analyzed by combining two concepts: beauty contest and moral hazard - a combination that allows for a translation of their behavior into the terms and the tradition of observation theory. Keynes' beauty contest can be interpreted as a systematic recognition of second-order observation: financial operators primarily observe other observers and what they observe. This observation produces particular circularities - including the insoluble problem of moral hazard, which reproduces Merton's famous model of self-defeating/self-fulfilling prophecies in the field of finance. If finance consists in secondorder observation, however, then its movements can no longer be explained in reference to the world, but in reference to observation and its structures: the reality reference of finance is increasingly provided by ratings, which can only offer information concerning what others observe. The spread of ratings in

REVISTA MAD - UNIVERSIDAD DE CHILE

Revista del Magíster en Análisis Sistémico Aplicado a la Sociedad

Facultad de Ciencias Sociales, Departamento de Antropología.

Universidad de Chile

www.revistamad.uchile.cl 
recent decades and the doubts about their reliability are related to the generalized move of modern society to second-order observation, which produces specific problems and specific puzzles, as well as structures and constraints.

KEYWORDS: Observation Theory; Embeddedness; Performativity; Ratings; Finance; Beauty Contest; Moral Hazard

\section{1. ¿OBSERVACIÓN EXTERNA DENTRO DE LA ECONOMÍA?*}

¿Cómo deberíamos describir hoy en día la condición de los sociólogos que observan procesos económicos? ¿Es posible observar la economía desde el exterior, y dónde estaría localizado tal exterior? Esta pregunta tiene implicaciones prácticas considerables, sobre todo cuando se describen procesos abstractos y reflexivos, como los movimientos financieros recientes, en los cuales las observaciones y las descripciones se mezclan directamente con operaciones de mercado, y en los que una sensibilidad sociológica específica puede ofrecer una mayor contribución. La reflexividad, descubierta con énfasis por la economía ${ }^{1}$, ha sido siempre uno de los supuestos de la observación sociológica. Hoy, los desarrollos teóricos disponibles nos permiten dar a estas preguntas una respuesta más compleja y eficaz que aquella que ha dirigido la investigación durante varias décadas pasadas.

Estas preguntas han sido por mucho tiempo relativamente no problemáticas. La respuesta clásica de Max Weber, así como está formulada incluso en el título de "Economía y Sociedad" (1922), pareció ser razonablemente suficiente: economía y sociedad son dos áreas distintas e interconectadas. Según este enfoque, la economía era entendida como un campo particular de instituciones y comportamientos que siguen sus propias reglas y criterios, y fuera de ella se ubica el campo más amplio de la sociedad en su conjunto. A fin de estudiar suficientemente la economía y sus procesos, se tienen que considerar también las relaciones entre las dos áreas y sus restricciones. La racionalidad económica, por ejemplo, no puede ser analizada abstractamente, es decir, aislando a la economía de aquellas influencias provenientes de estructuras sociales externas. Se debe conectar siempre el interior (economía) con el exterior (sociedad) y ver cómo comportamientos aparentemente irracionales a menudo son motivados

\footnotetext{
* [N. del T.] Traducido del inglés por Hugo Cadenas, con autorización de la autora. Una versión previa de este artículo apareció en: Sociologica, N², 2013.

${ }^{1}$ Aparte de Soros (1987), este es también el caso de todo el campo de la economía de la información (Stigler 1961; Stiglitz 1985, 2003; Grossman 1989) o de la "psico-socio-antropo-economía" de Akerlof (1984).
} 
por consideraciones extrañas a la lógica puramente económica, los cuales son empero significativos y en absoluto arbitrarios. De acuerdo con la conocida formulación de Granovetter (quién no por casualidad se refiere a Weber), "el comportamiento y las instituciones a ser analizados están tan constreñidos por las relaciones sociales en curso, que interpretarlos como independientes constituiría un penoso malentendido" (Granovetter 1985: 482).

Esta 'incrustación' [embeddedness] es dada por sentado hoy en día en la sociología económica y ha dado pie a numerosas y provechosas investigaciones. Una versión reciente, difundida bajo la etiqueta de teoría de la performatividad ${ }^{2}$, ha conducido una serie de iluminadores estudios sobre los mercados financieros y su dinámica, y ha ayudado también a identificar dificultades aparentemente insolubles que implicaban el problema de la observación de la economía y la posición del observador (Esposito 2013). La teoría de la performatividad comienza notoriamente del supuesto que la economía no consiste en una observación de carácter externa y neutral acerca de procesos económicos. La economía contribuye a la producción de la realidad económica que describe: sus aseveraciones y sus modelos "realizan, sacuden y dan formato a la economía" (Callon 1998: 2) y tienen efectos muy concretos en su dinámica. La teoría no es una observación externa, sino que está inevitable y necesariamente dentro del objeto de estudio, sea o no consciente de este hecho.

Esto nos obliga inmediatamente a plantear otra pregunta: ¿Dónde está ubicado el observador? ¿El sociólogo está dentro o fuera de la economía? ¿La sociedad, a la cual su observación pertenece, está dentro o fuera de la dinámica económica? En términos de Callon (2007: 316): “ ¿Cómo puede un discurso estar fuera de la realidad que describe y participar simultáneamente en la construcción de aquella realidad como un objeto que actúa sobre ella?" Una respuesta a esto ha sido buscada en la distinción entre "economía confinada" y "economía en la naturaleza"; ubicando a los sociólogos en un contexto más amplio que incluye otras disciplinas, manejos y prácticas (Callon 2007: 336). Sin embargo, siguiendo este camino la teoría sociológica que trata con la economía se convierte también entonces en una forma de 'economía' y debería estar sujeta, tanto a la eficacia como a las constricciones de la performatividad.

En esta situación, la distinción clásica entre economía y sociedad se hace cada vez más difusa y nos movemos inadvertidamente hacia una

\footnotetext{
${ }^{2}$ Así en Callon (1998). Cf. MacKenzie (2006,2009), MacKenzie D., Muniesa F., Siu L. (2007), Callon M., Millo Y., Muniesa F. (2007).
} 
condición en la cual el observador (cada observador) se encuentra dentro de la sociedad que él describe y actúa con sus observaciones -una sociedad que incluye comportamientos económicos y que nunca puede ser observada desde el exterior. La teoría de sistemas de Niklas Luhmann habla de "autología" de la teoría sociológica (Luhmann 1997: 16 y ss.), en la cual cada observación, incluso la sociológica, pertenece a la sociedad -es decir a su objeto- y produce efectos sobre ella. Una teoría autológica tiene que tomar esto en cuenta y ser consciente que es incapaz de controlar sus efectos.

Lo que proponemos aquí es un cambio de perspectiva en la observación sociológica de la economía. En este contexto, la distinción relevante ya no es entre sociedad y una economía que estaría más o menos fuertemente incrustada en ella, sino más bien entre perspectivas de observación diferentes, específicamente entre observación de primer y segundo orden (von Foerster 1981, Luhmann 1990: 68 y ss.; 1991: 23 y ss.). Esta distinción ha mostrado ser iluminadora para describir dinámicas sociales.

La observación de primer orden es la de un observador que observa objetos en el mundo. Los observadores de segundo orden observan observaciones -en efecto, observan cómo observan los observadores. Pero la realidad social no está constituida de esta manera: no se compone de objetos u observadores aislados. Para describir lo social se debe describir cómo estos observadores se observan unos a otros, y observar que los otros observadores también observan observaciones. Esto trae consigo un nivel reflexivo que abre el camino hacia la complejidad indeterminada: no solo la referencia a un cuarto, quinto o enésimo orden de observadores que observan observaciones de órdenes más altos, sino también (y esto es empíricamente el caso más relevante) a la situación en la cual el observador que observa la observación de otros, resulta ser él mismo observado por otros como un observador, activando de esta manera una dinámica recursiva que es muy difícil de controlar (así como de describir). Cada uno pertenece al mundo como un observador observado por otros, a los cuales uno tiene la intención de observar.

La primera consecuencia es que en este nivel cualquier referencia al mundo entrega verdaderamente pocas instrucciones. En el entrelazamiento circular de observaciones se pierde cualquier referencia a los objetos. En la observación de observadores que observan a observadores uno no presta atención a cómo es el mundo: el mundo es el resultado, no la premisa del entrelazamiento de observaciones y no puede, por lo tanto, explicarlas. Al igual que von Foerster (1981) quien hizo hincapié sobre esto en reitera- 
das ocasiones, esto no significa que no hay una realidad o que la realidad no es importante -al contrario, él señala con astucia que no niega la realidad; incluso afirma que hay muchas realidades, pues hay muchos observadores que afrontan el mundo siempre de manera absolutamente noarbitraria (nadie inventa la realidad a su antojo), pero siempre en relación con una perspectiva específica.

En el presente artículo aplicaré este enfoque al caso específico de la reflexividad económica y mostraré cuáles son las ventajas que ofrece para explicar cómo y por qué la economía ha evolucionado hacia una abstracción cada vez mayor de sus criterios y sus operaciones (expresado por las finanzas), abandonando cualquier referencia al mundo y sus datos. Las operaciones de las finanzas se refieren principalmente a las operaciones de las finanzas y así desarrollan criterios circulares y autorreflexivos. Pero ¿por qué sucede esto? ¿Qué tipo de estructuras se desarrollan? ¿Cómo se relaciona esto con la evolución general de "la sociedad de riesgo" (Beck 1986, Luhmann 1991) y sus estructuras?

En los siguientes párrafos articularé y especificaré esta interpretación radicalizada de la inclusión del observador en la realidad que observa. El comportamiento de los agentes financieros puede ser descrito mediante la combinación de dos conceptos: el concurso de belleza y el riesgo moral -y de este modo puede ser traducido a los términos y tradición de la teoría de la observación. El concurso de belleza de Keynes puede ser interpretado como un reconocimiento sistemático de la observación de segundo orden: los operadores financieros observan principalmente a otros observadores y lo que ellos observan (sección 2). Esta observación produce circularidades particulares, debido al hecho que uno no puede observar la realidad desde fuera de la sociedad (sección 3). Si las finanzas se fundamentan en la observación de segundo orden, sin embargo, sus movimientos no pueden ser explicados con referencia al mundo, sino a la observación y sus estructuras: la referencia a la realidad de las finanzas es proporcionada cada vez más por calificaciones crediticias que solo pueden ofrecer información acerca lo que otros observan (sección 4). Esta dependencia de la observación produce problemas y enigmas específicos que se derivan del hecho de que la sociedad moderna se ha movido -en todos sus ámbitos- desde la observación de primer orden a la observación de segundo orden (sección 5). Las tendencias inexplicables en el mundo financiero pueden estar conectadas con la circularidad de la observación. 
Después de algunos años de reflexión, la aparentemente inextricable maraña de movimientos esotéricos de las finanzas ha comenzado a parecer más inteligible -aunque no más controlable. Con todas sus complicaciones, el estado actual del mundo financiero puede ser descrito como el resultado de la combinación de dos conceptos, los cuales son muy conocidos y citados, aunque a menudo han sido simplificados y en parte malentendidos. Estos dos conceptos no suelen aparecer combinados.

El primer concepto es el famoso 'concurso de belleza' descrito por Keynes, una alusión que ha estado circulando durante muchas décadas en las reflexiones sobre las finanzas: la analogía de las finanzas como un concurso de belleza, en el cual el jurado debe elegir a las mujeres más bellas a partir de una muestra de fotografías -el ganador es quien adivina qué caras fueron las más votadas por los miembros del jurado. El problema, y el mayor estímulo para las observaciones de las finanzas, es determinar los criterios que un jurado prudente habría seguido:

No es el caso de seleccionar aquellas que, según el mejor juicio propio, son realmente las más bellas, ni siquiera las que la opinión general cree que lo son efectivamente. Hemos alcanzado el tercer grado en el que dedicamos nuestra inteligencia a anticipar lo que la opinión promedio espera que sea la opinión promedio. Y existen algunos, según creo, que practican los grados cuarto, quinto y otros superiores." (Keynes 1936: 156).

Aparentemente se trata de un simple caso de incrustación: no basta con observar el comportamiento como si fuese independiente de las relaciones sociales, es decir, de las conexiones con otros operadores. No basta con orientarse por criterios de belleza abstractos. Hay que considerar lo

\footnotetext{
* [N. del T.] Citado de acuerdo con la traducción de Eduardo Hornedo. Ver: Keynes, J. M. (1965). Teoría general de la ocupación, el interés y el dinero. México D.F.: FCE, pp. 142-143.

${ }^{3}$ Este es realmente un curioso concepto, mucho más apropiado para describir el mundo de las finanzas que la real situación de un concurso de belleza, donde el ganador no es el miembro del jurado más inteligente, sino una de las chicas concursantes -quiénes, por su parte, no tienen ningún papel en el modelo de Keynes. En las finanzas, la realidad de referencia es creada por las expectativas mutuas de los operadores y los concursantes son irrelevantes -como un concurso de belleza en el cual las muchachas son el resultado de las expectativas de los miembros del jurado (una imagen en muchos aspectos desagradable). Sin embargo, el modelo keynesiano del concurso de belleza es tan importante y se encuentra tan establecido en la descripción de los movimientos de las finanzas, que lo tomaré como referencia a pesar de sus defectos -que a su vez se benefician de la dinámica auto-referencial de los mercados. Para la aclaración de estos puntos me han sido de mucha ayuda las discusiones durante la Conferencia "Embeddedness and Beyond", Moscú 2012: Quisiera agradecer a los participantes, especialmente a David Stark y Flaminio Squazzoni.
} 
que los otros piensan. Pero aquí, si se abandona cualquier referencia a criterios 'objetivos' (del mundo exterior) y uno se aventura en el campo de las opiniones y las estructuras sociales, la situación se hace inmediatamente mucho más compleja: no basta con referirse a lo que los otros piensan, hay que referirse ahora a lo que los otros piensan que los otros piensan. Se trata de una observación de segundo orden, en el sentido descrito previamente.

El resultado del concurso de belleza no puede ser explicado discerniendo quién es la muchacha más hermosa. Esto no es lo que los miembros del jurado deben observar si quieren ganar el concurso. Hay que observar a los observadores y cómo ellos observan su mundo: observar lo que los otros piensan de las caras de las muchachas y determinar la opinión promedio. Pero incluso esto no basta: los observadores saben que no están solos y que los otros miembros del jurado también observan las observaciones de los otros y determinan la opinión promedio. Esto motiva la pregunta de ¿cómo aparecerá la opinión promedio de acuerdo con sus puntos de vista? Habría que ser capaz de observar cómo los otros lo observan, es decir, una especie de opinión promedio de la opinión promedio, moviéndose al tercer grado señalado por Keynes. Una vez que uno llega a este nivel es difícil decir dónde habría que detenerse, ya que esta opinión también puede ser observada en órdenes de observación más altos y más altos: cuarto, quinto, etcétera.

El aspecto interesante (y empíricamente relevante), sin embargo, es que esta regresión no conduce a una situación indeterminada donde la observación pierde toda referencia -donde la realidad ya no tiene fuerza vinculante. En el concurso de belleza la referencia a las observaciones de los otros no es arbitraria: se puede ganar o perder y uno gana si ha observado correctamente. No obstante esta precisión descansa sobre otros criterios que son mucho más complejos que la simple referencia a la belleza de las muchachas. La metáfora, debemos recordar, concierne a las finanzas: indica que el mundo financiero se guía por criterios precisos, y no arbitrarios, que no tienen nada que ver con la calidad real de los bienes o la solidez de las compañías -o con otros presuntos 'fundamentos' de la economía. Estos existen y son relevantes pero solo atañen a la observación de primer orden. No conducen la dinámica de las operaciones, las cuales se centran más bien en la observación de segundo orden, en la mutua observación de la observación de los otros, y esto ocurre hasta muy altos y aparentemente inextricables niveles de abstracción y circularidad. Es hacia es- 
to último que debiera dirigirse la atención de los estudiosos (y los sociólogos).

\section{EL RIESGO MORAL Y LAS PROFECÍAS AUTOFRUSTRADAS}

A fin de describir adecuadamente la complejidad del mundo financiero, nuestra referencia al concurso de belleza debe ser combinada con otra, la cual ha sido también muy observada y discutida en los últimos tiempos: la evasiva noción del riesgo moral. Esta noción introduce un momento de reflexividad que bloquea la continua regresión de cada orden de observación hacia uno más elevado: la condición en la cual el propio observador de segundo orden es observado por los observadores que él observa. De este modo, su propia observación afecta el mundo que él quiere describir.

El concepto de riesgo moral, desarrollado originalmente en el campo de los seguros, se ha extendido ahora a todos los aspectos del comportamiento económico, desde el nivel microeconómico de la teoría del contrato y las decisiones individuales, hasta la política económica y financiera. El riesgo moral indica que las consecuencias de una decisión afectan la situación a la cual la decisión se refiere, cambiando sus resultados y limitaciones: por ejemplo, los bien conocidos casos en los cuales una persona asegurada está mucho más dispuesta a asumir riesgos que aquellos que no están asegurados. El seguro que debería proteger contra riesgos, genera nuevos riesgos que no puede controlar (o solo puede hacerlo con gran indeterminación), porque el mundo en el cual los seguros actúan es diferente del mundo no asegurado que el asegurador es capaz de observar. Lo mismo ocurre en la política económica que siempre debe tener en cuenta la forma en que los mercados financieros reaccionan a las medidas reguladoras, por lo general saboteándolas con comportamientos que disminuyen de antemano los efectos de tales medidas. Por ejemplo, el Banco Central Europeo adoptó recientemente precauciones de no tomar decisiones de apoyo a las economías nacionales públicas ${ }^{4}$, sabiendo que el solo efecto de dicho anuncio iba a cambiar las condiciones de los mercados $\mathrm{y}$, por lo tanto, la eficacia de las medidas.

Un mundo gobernado por el concurso de belleza, como las finanzas, es inevitablemente presa del riesgo moral, precisamente debido a la circularidad de la observación: el propio observador de segundo orden es observado como observador por los observadores que él observa y, por lo

\footnotetext{
${ }^{4}$ Ver las declaraciones hechas por Mario Draghi's entre los meses de agosto y septiembre de 2012.
} 
tanto, su comportamiento afecta el mundo que observa. En otras palabras: en el nivel de la observación de segundo orden (que es, como sabemos, el nivel de las finanzas) uno no puede ver el mundo desde fuera. La observación de la realidad afecta la realidad que se observa, produciendo sorpresas y efectos inesperados -no porque el observador observe de manera imperfecta o descuide algunos factores, sino precisamente porque su comportamiento produce factores adicionales que antes no existían y no podían ser observados. Las consecuencias del comportamiento de los observadores son siempre parcialmente sorprendentes, aun cuando no son en absoluto aleatorias. Ellas dependen estrictamente de lo que se ha hecho: la realidad enfrentada es diferente si se hace algo o se hace nada. La realidad de los observadores resulta de su observación y, por lo tanto, suele ser diferente de lo que se esperaba.

El concepto de 'selección adversa', que se relaciona a menudo con el de riesgo moral, describe esta constelación: una condición de crónica carencia de información, debido a que la información relevante se produce por el propio comportamiento de los actores implicados en la decisión y no existe de antemano. Los accionistas de una compañía no pueden saber si un gerente está en condiciones de dirigir antes de probarlo y, por lo tanto, deben tomar su decisión sin la información crucial destinada en último término a conducir dicha decisión -y ningún acopio adicional de información les permite llenar este vacío de conocimiento.

Para un sociólogo, no obstante, esta enigmática condición no representa sorpresa. Uno de los pensadores clásicos de la disciplina, Robert K. Merton, la describió hace varias décadas atrás, en el contexto de una discusión sobre profecías autocumplidas y autofrustradas (Merton 1936, 1968: 477). Las predicciones y observaciones, como señala Merton, no contribuyen, como se podría pensar, a la determinación del futuro, sino que por el contrario lo hacen cada vez más imprevisible -ellas contribuyen, en términos de Merton, a perpetuar un "régimen del error". Si se cumplen las predicciones es porque a menudo se parte de una definición falsa (por ejemplo, de una descripción poco realista de los mercados ${ }^{5}$ ) que modifica la situación sobre la que se actúa, provocando comportamientos que la hacen verdadera si se la mira en retrospectiva -haciendo creer a quién enunció la definición que esta era verdadera desde un principio. No osbtante, cuando no se cumplen las predicciones, como es el caso de las profecías autofrustradas, la definición inicial suele ser verdadera (o al me-

\footnotetext{
${ }^{5}$ El famoso caso de "performatividad" descrito por MacKenzie (2006, MacKenzie \& Millo 2003) con referencia a la fórmula Black-Scholes para las opciones de precios.
} 
nos correcta sobre la base de la información disponible), haciéndose falsa solamente porque ha sido comunicada. De hecho, mientras más correctas son la predicción y la estrategia consiguiente, estas se vuelven más convincentes y serán seguidas, mostrándose eficaces en última instancia en la modificación de las condiciones sobre las cuales se basan, es decir, falseándose a sí mismas. Una falsa predicción tiende luego a hacerse verdadera debido a que fue comunicada y se refuerza por medio de interpretaciones posteriores, mientras que una predicción verdadera tiende a autofalsearse y producir una situación de incertidumbre y desorientación.

En términos de la reciente teoría de la performatividad, podría decirse que la observación de la economía siempre es performativa, en el sentido que tiene efectos en el mundo pero no puede controlarlos -en efecto, la performatividad conduce a menudo a confirmar observaciones falsas y a descartar las correctas (produciendo los bien conocidos efectos contraperformativos y también fenómenos como el "riesgo modelo" (Rebonato 1999)). Yendo aún más lejos, debemos señalar que la performatividad en sí puede ser observada. Un observador puede observar como la observación mutua entre agentes en el mercado produce efectos de resonancia (Beunza y Stark 2012: 410) que adoptan la forma de una burbuja y que pueden funcionar en oposición, "explotando la resonancia" para obtener ganancias. Casos bien conocidos, como el de John Paulson, el administrador de fondos de cobertura que obtuvo 15 mil millones de dólares yendo en contra de las tendencias del mercado durante la burbuja de 2008 (Zuckerman 2009), no revelan necesariamente una capacidad mayor para la evaluación de los fundamentos de la economía (Paulson mismo era relativamente un aficionado en materia de bienes raíces), sino la plausibilidad del movimiento hacia un orden de observación más elevado: desde la observación de otros observadores, hacia la observación de la observación misma como un hecho que afecta al mundo -y por lo tanto prestando la atención necesaria para aprovechar el momento adecuado y desviarse de la tendencia performativa, para luego tomar ventaja de los resultados. En la reconstrucción que hace Zuckerman (2009), el éxito de Paulson no se basó simplemente en uno de aquellos innumerables intentos por navegar en medio de una locura especulativa, las cuales son evidentemente muy peligrosas y donde él no estaba solo. Paulson hizo algo más: estuvo dedicado activamente a producir una deuda aún más tóxica, contra la cual él podía luego apostar (Zuckerman 2009: 179 y ss.), es decir, a intervenir performativamente en la observación de actores, manteniendo una posición independiente. Bajo estas condiciones, un nivel de no partícipe (es 
decir, el estatus de un operador no muy experimentado) puede ser también una ventaja ${ }^{6}$. Es, en cierto modo, una observación externa -externa a la observación de otros operadores, pero no externa a la economía. Incluso esta perspectiva será posteriormente observada, es decir interiorizada, y no puede garantizar una ventaja permanente.

En general, se puede asumir que en tiempos de alta incertidumbre la atención tiende a moverse desde la observación de primer orden a la observación de segundo orden: uno observa lo que los otros hacen en lugar de observar cómo las cosas son. Cuando el mundo se hace poco fiable, no es suficiente con observar el mundo. En efecto, mientras más aumenta la volatilidad del mercado, más abstracta e indirecta se hace la observación, hasta llegar a observar lo que los otros hacen, no para hacer lo mismo que ellos, sino para actuar de un modo diferente -para no conformarse, sino desviarse. Esto explicaría la emergencia de una misteriosa "asimetría de volatilidad" durante la crisis financiera reciente: una condición en la cual algunos operadores aparentemente creyeron que era más probable que ocurrieran los eventos improbables (Mandelbrot \& Hudson 2004, Esposito 2011: 148 y ss.). Esto no representa simplemente un caso de arbitrariedad de las tendencias financieras: el cisne negro* no revelaría simplemente el papel irreprimible del azar (Taleb 2001, 2007), sino más bien la capacidad de los observadores para observar la dependencia de los cálculos y modelos probabilísticos que presentan los mercados, obteniendo información, no de cómo marcha el mundo efectivamente, sino de lo que los demás esperan. La improbabilidad de los acontecimientos en juego sigue siendo absoluta con respecto al mundo y mucho menor con respecto a los observadores. En condiciones de performatividad, esta información es muy valiosa a fin de imaginar lo que podría suceder de una u otra forma (Esposito 2013).

Ampliando la atención desde la sola observación, hacia el contexto global y, por último, hacia la sociedad en su conjunto, la teoría de la performatividad (y la teoría de la observación) puede escapar de esta circularidad paralizante y proporcionar orientaciones sobre el funcionamiento general de la economía y las finanzas. En los términos de Keynes: hay que moverse hacia el cuarto o quinto nivel de observación, aquellos donde un

\footnotetext{
${ }^{6}$ Pero de ninguna manera es una garantía: Zuckerman (2009) muestra cómo muchos otros operadores sin experiencia sufrieron grandes pérdidas. No es suficiente con estar fuera, uno tiene que ser capaz de estar adentro y afuera al mismo tiempo.

" [N. del T.] La autora se refiere a la teoría del 'cisne negro' del matemático Nassim Nicholas Taleb (2001, 2007), según la cual existen eventos históricos que poseen un gran impacto y causan gran sorpresa, cuyas causas se analiza posteriormente como el resultado de una situación esperable.
} 
observador que sabe que es observado y toma este hecho en consideración, formula una teoría en la cual el observador mismo es parte del objeto que observa y está sujeto a las mismas limitaciones que se propone estudiar.

\section{LA REALIDAD DE LAS FINANZAS COMO REALIDAD DE CALIFICACIONES CREDI- TICIAS}

Si la realidad de las finanzas es el resultado conjunto del concurso de belleza y del riesgo moral, ¿qué consecuencias pueden ser trazadas y que instrumentos teóricos se requieren?

En primer lugar, la teoría de las finanzas debe estar ubicada siempre en el nivel de la observación de tercer o cuarto orden: para explicar los movimientos de las finanzas no hay que comenzar desde la solidez y la eficacia de compañías o desde la fiabilidad de los bancos, sino de la forma en que se observan estos factores en las finanzas y sus operaciones. Hay que observar entonces a observadores que observan a observadores. ¿Pero cómo se puede observar lo que otros observan? La teoría de la observación parte de la premisa que los observadores individuales son cajas negras unos para otros (von Foerster 1972); la teoría de sistemas sociales fue formulada para describir cómo estructuras sociales confiables y (hasta cierto punto) compartidas, se desarrollan a partir de una nebulosa de sistemas psíquicos mutuamente inaccesibles (la famosa y polémica aseveración de Luhmann que los pensamientos de las personas no pertenecen a la sociedad: Lumann 1997: 24 y ss.). Nunca es posible entrar en la mente de los demás, ni siquiera es necesario a fin de lograr observaciones de segundo orden.

La observación individual no es directamente observable, pero puede ser observada indirectamente a través de la comunicación. Esto no ocurre debido a que la comunicación expresa exactamente lo que el emisor tiene en mente (todos conocemos la distancia entre lo que pensamos y lo que podemos o queremos decir) ni porque el receptor entiende el sentido exacto de lo que se comunica (una comunicación exitosa siempre implica un malentendido: cada uno entiende la comunicación de manera diferente), sino simplemente porque la comunicación permite observar lo que otros observan y este nivel de participación es más que suficiente. Uno no necesita saber cómo ellos observan. Piénsese otra vez en el concurso de belleza de Keynes: el mecanismo funciona si los miembros del jurado tienen a su disposición algún instrumento para observar lo que los otros ob- 
servan, mientras que para ganar el concurso resulta totalmente irrelevante cuál muchacha fue considerada como la más hermosa por cada uno, así como lo que cada jurado realmente piensa. La opacidad de los pensamientos individuales, además de ser una garantía para la libertad y autodeterminación de los individuos, no representa un obstáculo para la construcción de estructuras sociales compartidas: uno no necesita saber lo que los otros piensan, sino lo que observan.

La sociedad, entonces, debe adoptar formas que permitan a los observadores observar las observaciones disponibles entre sí y trazar una orientación (ya sea conformidad o desviación). Cuando aumenta la complejidad, la referencia al mundo ya no es suficiente: se requieren herramientas que permitan observar lo que los otros (a su manera) observan. En el caso de las finanzas, estos instrumentos son principalmente las evaluaciones de las agencias de calificación crediticia. La realidad de las finanzas, a la cual se refieren los operadores financieros y hacia la cual orientan sus decisiones, no es más la realidad de primer orden de los presuntos 'fundamentos' de la economía, ni es tampoco la realidad de sus reflexiones sobre los mercados y sus movimientos: es la realidad mediada y compartida proporcionada por las agencias de calificación. Por lo menos desde los años setentas, la realidad de las finanzas es la realidad de las calificaciones crediticias. Las calificaciones indican a los operadores financieros (a los emisores y compradores, a los especuladores e inversionistas y cada vez más también a los reguladores) qué observar y cómo, los movimientos y tendencias en los mercados - a partir de una curiosa pero no arbitraria manera basada en una supuesta observación 'objetiva' del riesgo crediticio.

El movimiento desde la observación de los mercados a la observación de las calificaciones crediticias (que observan mercados) es un fenómeno evidente, extendido, pero aún en gran medida obscuro: "no hay duda que las agencias de calificación crediticia están entre las instituciones financieras más poderosas y menos comprendidas del planeta" (Langohr \& Langohr 2008: 473). Es también un fenómeno relativamente reciente, unido a los turbulentos cambios que hicieron cada vez más abstractas y misteriosas a las finanzas de las décadas pasadas (Sinclair 2010). No es casualidad que la creciente importancia y tamaño de las agencias de calificación crediticia (ACC), las cuales existen desde principios del siglo veinte, coincida con el período que comienza a partir de los años setentas, en el cual el sistema financiero global se convirtió en uno basado en el mercado y se desplazó desde los tipos de cambio fijo a los tipos de cambio flexibles, 
desde el control de precios a los precios de mercado, y donde las técnicas de finanzas estructuradas se han extendido enormemente -es decir, los mercados se han vuelto mucho más complejos y el riesgo ha tomado un papel central. En estos mercados, cada vez más opacos e incontrolables, las ACC experimentaron una enorme expansión (desde 1976 hasta 2006 el negocio de las calificaciones crediticias se más que quintuplicó7) y se extendieron a todas las áreas; se han hecho cada vez más objeto de críticas, pero también se han vuelto cada vez más indispensables. Es una convicción generalizada que si ellas se suprimieran, tendrían que crearse instituciones análogas para sustituirlas y sería difícil lograr su misma eficacia (p. ej. Langohr \& Langohr 2008: 371).

¿Por qué es que necesitamos tal construcción mediada e indirecta? ¿Por qué los observadores no pueden simplemente observar los mercados y entender las tendencias en las finanzas y hacer inversiones? La respuesta a estas preguntas se deriva de las consecuencias de la constelación presentada anteriormente, de la combinación entre el concurso de belleza y el riesgo moral, es decir, de la circularidad inevitable de la observación de observadores. Cuando uno abandona toda referencia al mundo y se mueve hacia la referencia a los observadores ${ }^{8}$, ya no hay ninguna referencia única o independiente, aunque sigue habiendo una realidad que pone limitaciones y esto no ocurre en absoluto al azar. Estas limitaciones (que existen y permanecen) dependen de la observación mutua de observadores, es decir, de una realidad mucho más abstracta, circular y mediada. Se necesita una referencia que la haga observable de una manera confiable y compartida: esta es la tarea de las agencias de calificación crediticia.

La literatura y teoría económica describen la función de las ACC de manera diferente a la teoría de la observación, si bien a través de líneas similares. Ellas sostienen que las ACC realizan una función vital en estos opacos mercados: sin sus indicaciones sobre la fiabilidad de los créditos, por ejemplo, uno tendría que tener cuidado con cualquiera que ofrece un crédito; los buenos operadores serían perjudicados y los malos beneficiados -con el resultado de un aumento en el costo de financiamiento externo y una disminución en la oferta9 .

El problema sigue siendo la omnipresente asimétrica información. En la actualidad se reconoce ampliamente que los mercados de valores [security markets] son mercados de información, en los cuales se inter-

\footnotetext{
${ }^{7}$ Fuente: Moody's Investors Service 2007, Corporate Default and Recovery Rates, 1920-2006, Exibit 33.

${ }^{8}$ Esto es especialmente cierto bajo condiciones de alta incertidumbre.

${ }^{9}$ Un fenómeno análogo al que describe Akerlof (1970) para el mercado de los limones.
} 
cambia información en primer lugar (Stigliz 1985, 2003; Grossmann 1989). Según el modelo ideal de los mercados ${ }^{10}$, esta información debería estar alojada en los precios de los valores: un operador debería tener toda la información relevante al observar un mercado eficiente. El problema radica en que la información es una entidad muy esquiva que no puede ser intercambiada sin modificar su naturaleza ${ }^{11}$ y los observadores no pueden limitarse a observar precios, sino también deben observar la forma en que estos se comunican. Una información comunicada cambia, porque un observador puede también preguntar por qué fue comunicada y con qué intenciones. Los bienes intercambiados en mercados financieros se modifican en el curso de las operaciones comerciales. Hay, por tanto, una asimetría inevitable entre insiders y outsiders: los inversionistas no saben realmente lo que ocurre dentro de una compañía y ninguna norma de transparencia puede cambiar esto y llenar el vacío de información. Parte de la información no puede ser revelada a los outsiders sin cambiar su sentido: por ejemplo, en los casos en que las malas noticias sobre las perspectivas de una compañía se han extendido, los insiders pueden saber muy bien que esto es una condición transitoria o que su impacto será irrelevante. Sin embargo, no pueden comunicar este hecho, ya que el mercado (después de las malas noticias) no creería en sus afirmaciones. Este observaría a los insiders e inevitablemente preguntaría por qué están diciendo tales cosas, añadiendo a la información transmitida sospechas acerca de sus motivos. O, para ser creíbles, los insiders deberían entregar información confidencial significativa, la que no puede ser difundida sin poner en peligro los intereses de los inversionistas.

Las calificaciones crediticias deberían servir para "tender un puente sobre la asimetría de información entre insiders y outsiders" (Langohr \& Langohr 2008: 12 y ss., también Kessler 2007: 311), ocupando una posición intermedia entre el interior y el exterior, integrando la información incluida en precios (calificaciones de mercado implícitas) con información adicional que los precios no pueden expresar, pero que es necesaria a fin de evaluarlos e interpretarlos. Este sería el valor agregado que ofrecen las calificaciones crediticias y la razón por la cual un cliente debería estar dispuesto a comprar los servicios de las ACC -y de manera creciente, pues los mercados se han hecho más complejos y menos transparentes.

\footnotetext{
${ }^{10}$ Empezando con la hipótesis de la eficiencia del mercado de Fama (1970).

${ }^{11}$ Heinz von Foerster (1972) señaló hace algunas décadas que la información no es un hecho del mundo, sino que debe estar relacionada con los observadores: "El entorno no contiene información, el entorno es como es".
} 
Pero, ¿realmente cumplen esta función las calificaciones crediticias? ¿Pueden cumplirla? Las cada vez más extendidas críticas sobre la labor de las ACC y sus presupuestos arrojan dudas sobre esta construcción (Sylla 2002, Hilscher \& Wilson 2012). Es casi imposible evaluar el valor informativo de las calificaciones: a menudo se ha criticado que estas van a la zaga de los mercados, ya que ellos anticipan la mayor parte de los cambios en las calificaciones (Partnoy 2002, Kessler 2007: 315) y que estas fallan sistemáticamente en anticipar las crisis (Reinhart 2002). En realidad, se trata del caso vergonzoso de productos cuya calidad no puede ser evaluada antes de comprarlos: la calidad de las calificaciones crediticias solo es revelada ex-post, cuando se puede verificar la exactitud de sus predicciones (Langohr \& Langohr 2008: 407).

Esta precisión está aún lejos de ser unívoca. La indeterminación fundamental de las calificaciones crediticias consiste en que no se puede evaluar su eficacia para la orientación de los mercados. En muchos casos, los precios realmente tienden a corresponderse con los cambios en las calificaciones $\mathrm{y}$, por lo tanto, las confirman, pero esto puede ser debido al efecto de las propias calificaciones, las cuales afectan a los mercados y sus movimientos (Partnoy 2002). ¿Los movimientos de los mercados confirman las calificaciones debido a que sus predicciones eran correctas, o porque los mercados han cambiado debido a las predicciones de calificaciones? Se trata de una típica condición performativa ${ }^{12}$ que produce efectos procíclicos: razón por la cual las ACC han sido acusadas de ser "bomberos pirómanos" (p.ej. Alain Minc en enero de 2012) que crean los mismos problemas que deben luego solucionar.

Desde el punto de vista de la teoría de la observación, estos problemas de circularidad son una consecuencia inevitable de la ambigua posición de las calificaciones con respecto de las finanzas: su función debería descansar en su ubicación externa e interna en un momento dado, a fin de resolver la asimetría de información. Pero la inclusión del observador es inevitable: si él quiere poner sus observaciones a disposición de las finanzas, cualquier observador (por lo tanto también las ACC) puede intervenir en el circuito de observación que él observa, él mismo debe ser observado y esto tiene consecuencias. La pretensión de objetividad de las ACC depende de simular su funcionamiento dentro de las finanzas como si estuvieran fuera y como si pudieran proporcionar una medida objetiva de riesgo. No obstante, los mismos autores que defienden el papel informativo esencial de las ACC deben reconocer que "las calificaciones crediticias es-

\footnotetext{
${ }^{12}$ La llamada performatividad barnesiana: cf. MacKenzie (2006).
} 
tán destinadas a ser exógenas, en el sentido que son indicadores externos, pero al mismo tiempo la incrustación de las calificaciones en diversos contratos, normas y regulaciones implica que tienen efectos reales directos y tienden a hacerse endógenas" (Langohr \& Langohr 2008: 474).

\section{OBSERVANDO LO QUE LOS OTROS SABEN}

Las calificaciones crediticias solo pueden ser internas a las finanzas y por tanto no pueden ser neutrales - pero esto no significa que sean inútiles, arbitrarias o que podamos prescindir de ellas. La afirmación de que si no existieran tendríamos que inventar algo más para llevar a cabo su función y que nada podría asegurar que se haría igual de bien o mejor esta tarea, sigue siendo correcta y aceptable. No obstante, esta función no puede fundarse en ubicar a las calificaciones como observadores externos, lo cual es imposible. Las calificaciones poseen otra característica que las distingue de otros operadores "incrustados" en los movimientos de las finanzas: ellas son "muy visibles" para todos los operadores (Langohr \& Langohr 2008: 474). Observando calificaciones, los operadores pueden observar lo que otros operadores observan y tomar esto como referencia (Sinclair 2010: 5). Esta visibilidad, y el hecho que todos son conscientes de ella, permite que las calificaciones provean a los participantes del mercado de un estándar o lenguaje común para referirse al riesgo de crédito, lo cual es observable y verificable (Langohr \& Langohr 2008: 90).

Esto funciona sin importar si las calificaciones son correctas o no, es decir, independiente de la cuestión insoluble de su correspondencia con el mundo (su valor informativo indeterminable). Las calificaciones, como los teóricos afirman incesantemente, no ofrecen declaraciones sobre hechos sino solamente opiniones, no importa cuán complicadas y controladas estas sean -siguen siendo opiniones entre muchas otras (Langohr \& Langohr 2008: 474, 17 y ss.). Ellas son opiniones acerca de un objeto particularmente incierto, ya que se refieren al futuro probable de valores o emisores (Langohr \& Langohr 2008: xiii) -respecto de los cuales afirman, además, considerar exactamente los aspectos improbables: las pérdidas inesperadas. Aquí la circularidad es aún más desconcertante, teniendo en cuenta que los requisitos para hacer frente a pérdidas inesperadas también dependen de la eficacia de las medidas adoptadas para afrontar daños esperados, es decir, dependen de lo que se espera -ante un futuro que todavía no ha llegado y que depende de nuestro comportamiento actual (Smith \& Walter 2002). 
Estos aspectos temporales son complejos y difíciles de manejar (Esposito 2011). Las calificaciones crediticias, enfrentadas con un objeto tan huidizo y circular, proporcionan a pesar de todo una referencia: "anticipar lo que la opinión promedio espera que sea la opinión promedio", que era el enigma del concurso de belleza de Keynes. Los observadores en el mercado saben que no saben cómo son las cosas y que no pueden saber lo que los otros saben y piensan. Lo que tienen son opiniones -siempre internas, circulares e inciertas. Pero los observadores pueden saber lo que otros observadores observan a fin de formar sus opiniones: una referencia compartida y visible, una opinión que está disponible para todos y que todos saben que se sabe (aun si uno no sabe lo que piensan de ella los demás). Este es el 'estándar común' proporcionado por las calificaciones y funciona independientemente de su exactitud -la cual puede ser totalmente irrelevante: lo que importa es cómo las calificaciones son observadas por observadores sobre la base de la reputación y otros factores intangibles y no en su relación con una presunta realidad externa (que en realidad se adapta a las calificaciones, en lugar de a la inversa) ${ }^{13}$. Es sobre esta opinión, como sabemos, que depende el éxito en el concurso de belleza.

Las calificaciones crediticias señalan, para el caso de finanzas, una tendencia cada vez mayor al nivel de la sociedad en su conjunto: la dependencia creciente de evaluaciones en todos los aspectos de la realidad social -en la elección de restaurantes, libros, computadoras y cualquier tipo de compra (Blank 2007), así como la explosión de las auditorías en todos los sectores, desde las finanzas hasta la asistencia médica o la educación (Power 1997). En todos estos casos, hay un movimiento sistemático desde la observación de primer orden sobre datos (los restaurantes para visitar, los libros para leer) hacia la observación de segundo orden sobre la observación de los demás (lo que otros piensan del restaurante y del libro en cuestión). La elección, y por lo tanto la relación con el mundo, dependen de esto.

Nuestra sociedad tiende a expandir en todas las áreas el patrón bien establecido de los medios de comunicación de masas, los cuales construyen lo que se convierte luego en la realidad compartida por todos los ciu-

\footnotetext{
${ }^{13}$ Esto explica asimismo una paradoja adicional de las calificaciones crediticias (White 2002): el modelo "catch 22 " de las restricciones a la entrada en el mercado de las calificaciones. Los reguladores establecen criterios para proteger la calidad de las calificaciones y mejorar la competencia justa. Sin embargo, estos criterios no pueden concentrarse en el output (la eficacia de las predicciones sobre la probabilidad de cesación de pagos) sin considerar el input (la reputación de las empresas de calificación). El resultado es una condición en la cual una nueva firma no puede obtener reputación nacional sin ser reconocida como una organización de calificación con reputación nacional.
} 
dadanos. "Lo que sabemos sobre nuestra sociedad y en general sobre el mundo en que vivimos, lo sabemos a través de los medios de comunicación de masas." (Luhmann 1995: 9). La realidad a la cual todos nos referimos incluye lo que sabemos a través de la televisión, películas, libros y periódicos que no reportan fielmente la realidad, sino que construyen su propia realidad (al igual que las evaluaciones: Blank 2007. 4 y ss.) ${ }^{14}$, la cual solo debe ser creíble. Los medios nos proveen de lo que se convierte en la realidad disponible en la comunicación y en la vida social: conocimiento de lo que otros saben, al cual podemos referirnos y esperar ser entendidos, y que puede servir para una comunicación posterior (independientemente de lo que realmente crean o piensen los demás -como en el modelo del concurso de belleza).

La realidad de nuestra sociedad tiende a convertirse en la realidad de la observación de segundo orden (Luhmann 1997: 766 y ss.) -lo cual ayuda a explicar la tendencia similar y paralela, según la cual la realidad de la finanzas tiende a hacerse cada vez más la realidad de las calificaciones crediticias que no solamente dirigen las elecciones de operadores y compañías, sino que también conducen las decisiones de los reguladores. Los acuerdos de Basilea II, por ejemplo, utilizan las evaluaciones de las ACC para determinar las necesidades de capital de los bancos, provocando un movimiento circular adicional donde la regulación decide sus medidas a partir de sus resultados sobre la realidad a ser regulada (Esposito 2011: 165 y ss.).

\section{CONCLUSIONES}

Estudiar los movimientos de las finanzas de este modo plantea también diversos problemas -por ejemplo, cómo explicar la reciente disminución en la autoridad de las agencias de calificación crediticia, en particular en el caso de las emisiones soberanas. En el transcurso de 2012, las decisiones de S\&P y Moody respecto de las calificaciones crediticias de los Estados fueron a menudo ignoradas por los inversionistas, quienes fueron en dirección contraria. El descenso de la calificación de Francia en noviembre de 2012, por ejemplo, fue totalmente ignorado, incluso hasta contrarrestado por los mercados. Las decisiones del Banco Central Europeo durante el año pasado estuvieron totalmente desacopladas de las indicaciones de las ACC. Sin embargo, parece que los rendimientos de las

\footnotetext{
${ }^{14}$ La teoría de la elaboración de noticias lo mostró hace mucho tiempo: ver Altheide (1976), Altheide \& Snow (1979), Gans (1979).
} 
ACC siguen siendo esenciales: se siguen produciendo, difundiendo y comentando. ¿Cómo podemos explicar esta tendencia?

A partir de la decepción del caso Lehman Brothers, se ha extendido la idea respecto que las evaluaciones de las ACC son no confiables. Los observadores al parecer todavía necesitan una referencia compartida y las ACC son, por el momento, la única opción viable - aun si las formas en las que se utilizan están cambiando, en un marco de observaciones más complejas y articuladas. Es como si los mercados integraran perfomativamente las evaluaciones de las ACC y la observación relativa -uno observa ahora que los demás tampoco creen en ellas, sin embargo aún son observadas. Entonces el problema se desplaza: ¿Cómo observan los operadores las calificaciones de un modo negativo, a fin de observarse unos a otros? Habría que tratar de reconstruir la estructura performativa de esta observación, en la cual la referencia compartida actúa de manera más negativa que positiva: indica lo que los otros saben, a sabiendas que esto no es confiable. ¿Cómo utilizan los operadores esta información?

O mejor dicho: ¿observan todavía los operadores las calificaciones con el fin de orientar sus decisiones? En el momento en que uno se diera cuenta que los agentes ya no prestan atención a las evaluaciones de las ACC, su valor informativo colapsa: se convierten en opiniones como cualquier otra, sin el valor agregado que se desprende de su capacidad para indicar lo que los demás observan. Y luego habría que investigar qué tomaría su lugar: ¿Qué observan ahora los observadores con el fin de observar la observación de los demás (es decir, su realidad de referencia), si las calificaciones ya no son informativas? ¿Cómo se aborda la opacidad de los mercados a fin de evitar la arbitrariedad?

Estas son preguntas empíricas que se refieren a problemas complejos sobre los cuales no tenemos suficientes datos. Seguimos siendo incapaces de proporcionar una respuesta, sin embargo estos problemas hacen aún más evidente la necesidad de una cuidadosa y compleja teoría de la observación. El estudio de la incrustación de las finanzas en la sociedad requiere de instrumentos sofisticados que vayan más allá de la integración de la investigación sobre las finanzas con la referencia a la red de interacciones en las cuales los actores son incluidos: un estudio adecuado de las finanzas y sus peculiares estructuras circulares requiere, aquí como en muchos otros casos, una referencia a la sociedad.

Las herramientas para realizar este tipo de análisis pertenecen a la tradición de la sociología. La capacidad de teorizar la circularidad de la observación y sus efectos es el legado más fecundo de la clásica pregunta so- 
bre la incrustación en una sociedad y una economía que se han hecho cada vez más complejas. De hecho, la referencia a las finanzas y a la observación nos permite reformular el problema de la incrustación como una combinación entre el concurso de belleza (observación de observaciones) y el riesgo moral (circularidad de la observación). Desde este punto de vista, los aparentes enigmas de los movimientos de los mercados financieros se vuelven explicables -aun cuando ellos no puedan ser controlados. Esta interpretación podría extenderse al estudio de fenómenos económicos en general, moviendo la atención hacia las formas y condiciones de la inclusión del observador en el objeto que observa.

El movimiento de las finanzas hacia la observación de segundo orden y sus formas, como hemos visto, no puede ser analizado estudiando solamente la economía, ni siquiera teniendo en cuenta la performatividad y la referencia a los actores en las relaciones sociales. Corresponde a una transformación estructural de la sociedad en conjunto, la que tiene que afrontar un futuro abierto y la ubicuidad del riesgo -que se manifiesta de manera más urgente y significativa en las finanzas y sus incógnitas. La incrustación, si es útil, debe funcionar siempre en ambos sentidos, en efecto, con un mecanismo en espiral: la referencia a la sociedad sirve para explicar las estructuras de la economía, las que sirven luego para clarificar la evolución de la sociedad.RM

\section{BIBLIOGRAFÍA}

Akerlof, G. (1970). The Market for 'Lemons': Quality Uncertainty and the Market Mechanism. The Quarterly Journal of Economics, 84(3), 488-500. [Reimpreso en: Akerlof, G. (1984). An Economic Theorist's Book of Tales (pp. 7-22). Cambridge: Cambridge University Press.]

Akerlof, G. (1984). An Economic Theorist's Book of Tales. Cambridge: Cambridge University Press.

Altheide, D. (1976). Creating Reality. How TV News Distorts Event. Beverly Hills/London: Sage.

Altheide D. \& Snow R. (1979). Media Logic. Beverly Hills/London: Sage.

Beck, U. (1986). Die Risikogesellschaft: Auf dem Weg in eine andere Moderne. Frankfurt a.M.: Suhrkamp.

Beunza, D. \& Stark, D. (2012). From Dissonance to Resonance: Cognitive Interdependence in Quantitative Finance. Economy and Society, 41(3), 383-417.

Black, F. \& Scholes, M. (1981). The Pricing of Options and Corporate Liabilities. Journal of Political Economy, 81(3), 637-654.

Blank, G. (2007). Critics, Ratings and Society: The Sociology of Reviews. Lanham: Rowman \& Littlefield. 
Çalişkan, K. \& Callon, M. (2009). Economization, part 1: Shifting Attention from the Economy towards Processes of Economization. Economy and Society, 38(3), 369398.

Callon, M. (Ed.). (1998). The Laws of the Market. Oxford: Blackwell.

Callon, M., Millo, Y., \& Muniesa F. (Eds.). (2007). Market Devices. Oxford: Blackwell.

Esposito, E. (2011). The Future of Futures. The Time of Money in Financing and Society. Cheltenham: Elgar.

Esposito, E. (2013). The Structures of Uncertainty. Performativity and Unpredictability in Economic Operations. Economy and Society, 42(1), 102-129.

Fama, E. (1970). Efficient Capital Markets: A Review of Theory and Empirical Work. Journal of Finance, 25(2), 383-417.

Foerster, H. von (1972). Notes on an Epistemology for Living Things. BCL Report No.9.3, Biological Computer Laboratory, Department of Electrical Engineering, University of Illinois, Urbana. [Reimpreso en: Foerster, H. von (1981). Observing Systems (pp. 258-271). Seaside, CA: Intersystems Publications.]

Foerster, H. von (1981). Observing Systems. Seaside, CA: Intersystems Publications.

Gans, H. (1979). Deciding what's News. New York: Random House.

Granovetter, M. (1985). Economic Action and Social Structure: The Problem of Embeddedness. American Journal of Sociology, 91(3): 481-510.

Grossman, S. (1989). The Informational Role of Prices. Cambridge, MA: MIT Press.

Grossman, S. \& Stiglitz, J. (1980). On the Impossibility of Informationally Efficient Markets. American Economic Review, 70(3), 393-408.

Keynes, J. M. (1936). The General Theory of Employment, Interest and Money. London: Macmillan.

Kessler, O. (2007). Unsicherheit, Ungewissheit und Risiko: Temporalität und die Rationalität der Finanzmärkte. En A. Langenoh \& K. Schmidt-Beck (Eds.), Die Markt-Zeit der Finanzwirtschaft: soziale, kulturelle und ökonomische Dimensionen (pp. 293-321). Marburg: Metropolis.

Hilscher, J. and Wilson, M. (2012) Credit Ratings and Credit Risk. AFA 2013 San Diego Meetings Paper (January 31, 2012).

Langohr, H. \& Langohr, P. (2008). The Rating Agencies and Their Credit Ratings: What They Are, How They Work and Why They are Relevant. Chichester: Wiley.

Levich, R. Majnoni, G., \& Reinhart, C. (2002). Ratings, Rating Agencies and the Global Financial System. New York: Kluwer.

Luhmann, N. (1988). Die Wirtschaft der Gesellschaft. Frankfurt a.M.: Suhrkamp.

Luhmann, N. (1991). Soziologie des Risikos. Berlin/New York: de Gruyter.

Luhmann, N. (1992). Beobachtungen der Moderne. Opladen: Westdeutscher Verlag. Luhmann, N. (1995). Die Realität der Massenmedien. Opladen: Westdeutscher Verlag. Luhmann, N. (1997). Die Gesellschaft der Gesellschaft. Frankfurt a.M.: Suhrkamp.

MacKenzie, D. (2006). An Engine, Not a Camera. How Financial Models Shape Markets. Cambridge, MA: MIT Press.

MacKenzie D. (2009). Material Markets. How Economic Agents Are Constructed. Oxford: Oxford University Press.

MacKenzie D., Muniesa, F., \& Siu, L. (Eds.). (2007). Do Economists Make Markets? Princeton: Princeton University Press. 
MacKenzie, D. \& Millo, Y. (2003). Constructing a Market, Performing Theory: The Historical Sociology of a Financial Derivatives Exchange. American Journal of Sociology, 109(1), 107-145.

Partnoy, F. (2002). The Paradox of Credit Ratings. En R. Levich, G. Majnoni, \& C. Reinhart, C. (Eds.), Ratings, Rating Agencies and the Global Financial System (pp. 65-84). New York: Kluwer.

Power, M. (1997). The Audit Society. Rituals of Verification. New York: Oxford U.P.

Rebonato, R. (2001). Managing Model Risk. En C. Alexander (Ed.), Handbook of Risk Management. FT: Prentice Hall.

Reinhart, C. (2002). Sovereign Credit Ratings Before and After Financial Crises. En R. Levich, G. Majnoni, \& C. Reinhart, C. (Eds.), Ratings, Rating Agencies and the Global Financial System (pp. 251-268). New York: Kluwer.

Sinclair, T. (2010). Credit Rating Agencies and the Global Financial Crisis. Economic Sociology Newsletter, 12(1), 4-9.

Smith, R. \& Walter, I. (2002). Rating Agencies: Is There an Agency Issue? En R. Levich, G. Majnoni, \& C. Reinhart, C. (Eds.), Ratings, Rating Agencies and the Global Financial System (pp. 289-318). New York: Kluwer.

Soros, G. (1987). The Alchemy of Finance. Reading the Mind of the Market. Chichester: Wiley.

Stigler, G. (1961). The Economics of Information. The Journal of Political Economy, $69(3), 213-225$.

Stiglitz, J. (1985). Information and Economic Analysis: A Perspective. Economic Journal, 95, 21-41.

Stiglitz, J. (2003). Information and the Change in the Paradigm in Economics. En R. Arnott, B. Greenwald, R. Kanbur \& B. Nalebuff (Eds.), Economics in an Imperfect World. Essays in Honor of Joseph E. Stiglitz (pp. 569-639). Cambridge, MA: The MIT Press.

Sylla, R. (2002). An Historical Primer on the Business of Credit Ratings. En R. Levich, G. Majnoni, \& C. Reinhart, C. (Eds.), Ratings, Rating Agencies and the Global Financial System (pp. 19-40). New York: Kluwer.

Taleb, N. (2001). Fooled by Randomness. The Hidden Role of Chance in the Markets and in Life. New York/London: Texere LLC.

Taleb, N. (2007). The Black Swan: The Impact of the Highly Improbable. London: Penguin.

Weber, M. (1922). Wirtschaft und Gesellschaft. Tübingen: Mohr.

White, L. (2002). The Credit Rating Industry: An Industrial Organization Analysis. En R. Levich, G. Majnoni, \& C. Reinhart, C. (Eds.), Ratings, Rating Agencies and the Global Financial System (pp. 41-63). New York: Kluwer.

Zuckerman, G. (2009). The Greatest Trade Ever. The Behind-the-Scenes Story of How John Paulson Defied Wall Street and Made Financial History. New York: Broadway Books.

SOBRE LA AUTORA

Elena Esposito es profesora del Departamento de Comunicación y Economía de la Universidad de Módena y Reggio Emilia, Italia. Doctora en Sociología por la Universi- 
dad de Bielefeld, Alemania. Entre sus áreas de especialización se encuentran: Sociología de los Medios, Investigación sobre Memoria, Teoría de la Moda y Sociología Económica. Entre sus publicaciones se destacan: Il futuro dei futures. Il tempo del denaro nella finanza e nella società [ETS, 2009]; Probabilità improbabili. La realtà della finzione nella società moderna [Meltemi, 2008], I paradossi della moda. Originalità e transitorietà nella società moderna [Baskerville, 2004]; Soziales Vergessen. Formen und Medien der Gedächtnis der Gesellschaft [Suhrkamp, 2002]. En español: (Junto a Giancarlo Corsi y Claudio baraldi) GLU: Glosario sobre la teoría social de Niklas Luhmann [Universidad Iberoamericana, 1996]. Anteriormente en esta misma revista: "Los misterios del dinero" [Revista Mad - Universidad de Chile, N²29, 2013, pp. 26-34].

\section{CONTACTO}

Dipartimento di Comunicazione ed Economia Università degli Studi di Modena e Reggio Emilia viale Allegri 9, 42121 Reggio Emilia elena.esposito@unimore.it

Recibido: Octubre 2013

Aceptado: Enero 2014 